\title{
The pediatric gastrointestinal tract and ultrasonography
}

\author{
Atsushi Yoden
}

(C) The Japan Society of Ultrasonics in Medicine 2015

In recent years, ultrasonography has become increasingly precise in diagnosing abdominal and gastrointestinal diseases. In most cases, a definitive diagnosis for acute appendicitis, which is commonly encountered in daily practice, can be obtained by using ultrasound. In addition, hypertrophic pyloric stenosis and intussusception can be diagnosed with this device alone. Moreover, ultrasoundguided reduction of intussusception is widely used, making it possible to perform non-invasive reduction without radiation.

My first involvement with ultrasonography was 25 years ago, and hemorrhagic colitis caused by the $\mathrm{O}-157$ strain of $E$. coli is one of the memorable diseases from my experiences at that time. Unlike today, it was very common, and I had experienced about 10 sporadic cases of hemolytic uremic syndrome caused by the O-157 strain within a few years. Back then, the 5-layer structure of the gastrointestinal tract could not be observed in detail as it can be now. However, the walls of the colon thickened to such an extent that the lumen could be barely seen. In addition, when complicated by hemolytic uremic syndrome, the kidneys became hyperechoic and renal blood flow decreased within a few days. Culturing of the O-157 strain was very difficult, and many cases were diagnosed by preserving the serum of patients with unknown hemorrhagic colitis and checking the rise in antibody titer later.
A heavy annular array probe, used for examining breasts, was used to diagnose acute appendicitis, rather than the linear array probe used today. I was told that the sensitivity of the annular array probe was low for acute appendicitis, and luminal gas could not be observed in a normal appendix. After examining several dozen patients with acute appendicitis, however, I learned that even a normal appendix of a child sometimes has a diameter of more than $6 \mathrm{~mm}$, and that there are cases where luminal gas could be observed in a normal appendix. I also remember reading that the Kerckring folds were difficult to observe in a healthy child and could only be seen as the keyboard sign in intestinal obstruction. Today, the Kerckring fold can be easily observed in a healthy child.

The feeding vessels of the gastrointestinal wall can be observed in detail and ischemia can be easily evaluated, not only with color Doppler, but also contrast-enhanced ultrasound using Sonazoid. We have diagnosed ischemic enteritis of the small intestine using Sonazoid. Also, three-dimensional images of the gastrointestinal tract are starting to be constructed using ultrasound. Someday, 3D images will probably be easily made with ultrasound, and not only CT or MRI.

Keeping up with the rapid advances in ultrasound devices is becoming a task for sonographers. However, observation with the classical B-mode image in the transabdominal approach is fundamental, and so I hope that younger sonographers will understand its importance.
A. Yoden $(\bowtie)$

Department of Pediatrics, Osaka Medical College, Osaka, Japan

e-mail: ped018@poh.osaka-med.ac.jp 